\title{
Room temperature plasticity in thermally grown sub-micron oxide scales revealed by micro-cantilever bending
}

\author{
Anand. H. S. Iyer, Krystyna Stiller, Magnus Hörnqvist Colliander* \\ Department of Physics \\ Chalmers University of Technology \\ 41296-Gothenburg \\ Sweden \\ *Corresponding author: magnus.colliander@chalmers.se
}

\begin{abstract}
We propose a new geometry for focused ion beam milled micro-cantilevers, which allows production of residual stress-free, isolated thin film specimens from film-substrate systems. This geometry was used to demonstrate the presence of permanent deformation in about $200 \mathrm{~nm}$ thick thermally grown oxide scales on a Ni-base superalloy, after applying large bending displacements insitu in a scanning electron microscope. Stiffness measurements performed before and after the bending tests confirmed the absence of micro-cracks, leading to the conclusion that plastic deformation occurred in the oxide scale. The proposed method is extendable to other film-substrate systems and testing conditions, like non-ambient temperatures.
\end{abstract}

Keywords: Focused ion beam (FIB); scanning electron microscopy (SEM); crystalline oxides; plastic deformation; micro-mechanical testing.

The properties of protective oxide scales on engineering alloys play a crucial role in determining their service life in many applications. One example of this is the ability of oxide and underlying substrate to relax both growth stresses and thermal stresses to avoid scale cracking and spallation [1]. Other typical situations involve the reduced life during out-of-phase thermo-mechanical fatigue, where the oxides formed during the hot part of the cycle under compressive load undergo ductile-to-brittle transition and fracture during tension at low temperatures [2], and the repeated formation and fracture of oxide intrusions at crack tips during dwell-fatigue crack growth of superalloys $[3,4]$. In order to mitigate such problems, it is imperative to understand the mechanical properties of these scales under service conditions.

The mechanical properties of protective oxide scales have previously mostly been measured on macro-scale film-substrate systems using indirect techniques, such as acoustic microscopy and vibration technique [5,6], or by post-test inspection of the surfaces [7]. Even in the case of direct testing through nano-indentation, certain assumptions have to be made since the unloading curve is non-linear [6]. A critical point in such film-substrate testing is that the residual stresses and strains in the oxide scale are usually not well known, and the interpretation of measured fracture stresses and strains are therefore ambiguous. 
Oxide scales are generally considered to be brittle, and also typically contain inherent defects and residual stresses which lead to crack initiation and fracture [1,7]. Whereas plasticity has been observed in e.g. $\alpha-\mathrm{Al}_{2} \mathrm{O}_{3}$ [8] and $\mathrm{Cr}_{2} \mathrm{O}_{3}$ [9] at high temperatures, it is not expected to occur at room temperature, as below a transition temperature, the stress required for fracture is less than the flow stress [10]. Conventional indirect measurements of oxide scale behavior have traditionally been limited to thicker oxide scales, often in the range 5-50 $\mu \mathrm{m}$, in which the probability of defect occurrence is high. More recently, test methods using bi-layer beams extracted from macroscopic specimens have been used to measure in-plane elastic properties [11] and delamination toughness [12] of thermal barrier coatings. With the advancement of technologies such as focused ion beam (FIB) milling and micro-mechanical testing methods, the use of direct tests at the micro- to nano-scale, such as micropillar compression, has become possible [13-15]. Through such testing methods, a sizedependent transition from brittle to ductile behavior [16] has been shown for several material systems, such as metallic glasses [17], quasicrystals [18], sapphire [14] and silicon [13], which are brittle in bulk form. However, with few exceptions [17,18], testing has been performed in compression, which delays crack initiation and therefore promotes plastic deformation which would potentially be absent in other loading modes.

Here we present a novel method for measuring properties of thin films using micro-cantilever bending experiments applicable on sub- $\mu \mathrm{m}$ scale, and use this method to demonstrate the occurrence of room temperature plasticity during tensile loading of thermally grown oxide scales with thicknesses in the order of $200 \mathrm{~nm}$. In particular, the properties measured by the proposed method are not subject to influence from residual stresses, which can influence the results [7,11,19] and represent the isolated properties of the oxide scale, free of interactions with the substrate. Importantly, the method is applicable to different film-substrate systems, and is easily extendable to non-ambient temperatures, which is crucial for assessing the behavior during most service-like conditions. Nevertheless, the room temperature behavior of thermally grown oxide scales treated in this investigation is also of great interest in its own right, referring to e.g. out-of-phase thermo-mechanical fatigue, as mentioned above.

The oxide scales used in the present study were thermally grown on a solution treated and aged Allvac 718Plus superalloy (nominal composition in wt. \% Ni-18Cr-9.1Co-9.5Fe-5.4Nb-2.7Mo$1.45 \mathrm{Al}-1 \mathrm{~W}$ ) in dry oxygen environment at $700{ }^{\circ} \mathrm{C}$ for 100 hours, which resulted in an oxide layer about $200 \mathrm{~nm}$ in thickness (see supplementary material). The structure of the oxide was investigated using transmission electron microscopy (TEM) in a Titan 80-300 microscope operated at $300 \mathrm{kV}$. The oxide consisted of an outer spinel layer with a thickness of about $50 \mathrm{~nm}$, typically constituted by a single layer of spinel grains, followed by a chromia layer of about $150 \mathrm{~nm}$ thickness (Figure 1a). Below the surface scale, isolated internal alumina could be found. The EDS (energy dispersive X-ray spectroscopy) line scan in Figure $1 \mathrm{~b}$ shows the different elements present in the oxide layer, indicating a mixed $(\mathrm{Ni}, \mathrm{Co})(\mathrm{Fe}, \mathrm{Cr})_{2} \mathrm{O}_{4}$ spinel. While the cubic structure of the outermost part of the scale was verified by transmission Kikuchi diffraction, the details of the oxide scale has not been further studied at this stage. 

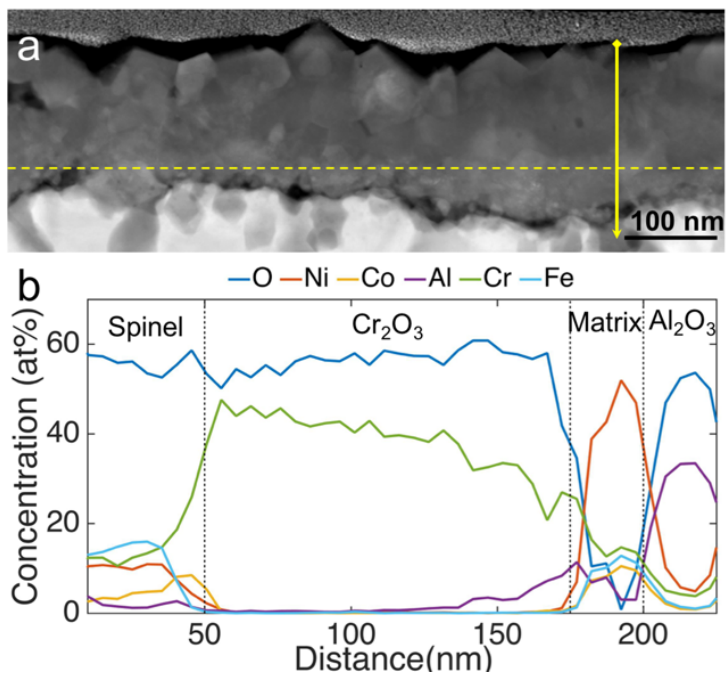

Figure 1: (a) TEM high-angle annular dark field (HAADF) image of oxide scale. (b) Results from TEM linescan showing the concentration of different elements in the scale. The vertical yellow line in (a) shows the position of linescan. The horizontal dotted line shows the position of the bottom of the cantilever after final specimen preparation (see text for more details).

Micro-cantilevers were prepared from the oxidized specimens using FIB milling in FEI Versa3D (see supplementary material for details). The geometry of the cantilevers (Figure 2) was designed in such way that the oxide layer was isolated towards the fixed end for about $2.5 \mu \mathrm{m}$ in length. This ensures that the residual stresses are relieved in this region, and that there is no metal underneath supporting the oxide layer. Attempts to produce longer oxide cantilevers without metal substrate failed due to the excessive bending from residual stress relaxation. Therefore, the metal was left below the remaining part of the cantilever, as it provided stable conditions for load applications, and the extended length acted as an amplifier for more accurate displacement measurements (see below). The dotted line in Fig. 1a shows the typical location of the final bottom edge of the finished cantilevers. As material was only removed from the bottom of the cantilever, minimal ion implantation was obtained at the top surface, which is the region subjected to tensile stresses in the tests.

The bending experiments were conducted in FEI Quanta 200 FEG ESEM scanning electron microscope (SEM) in high vacuum using a Kleindiek micromanipulator setup. The force was measured using a Kleindiek force measurement sensor (FMS), which provides a voltage signal from a bending piezo-electric beam used to apply a displacement to the cantilever. The FMS was calibrated against a copper spring with known spring constant (uncertainty of about $10 \%$ ), and the accuracy of the calibration procedure itself was determined to be in the order of $\pm 5 \%$. Displacements were measured through post-processing of images taken in the SEM at a frequency of $1 \mathrm{~Hz}$ during testing. A schematic of the experimental setup is shown in Figure 2a.

Initially, three tests were performed on micro-cantilevers with similar size (denoted A, B and $\mathrm{C}$ in Table 1, where all relevant dimensions are included). For each cantilever, a number of consecutive bending cycles were applied, with progressively increasing maximum displacement. The displacement of a point at the fixed end was measured to give an estimate of the drift during testing, which was negligible in all tests reported herein. 

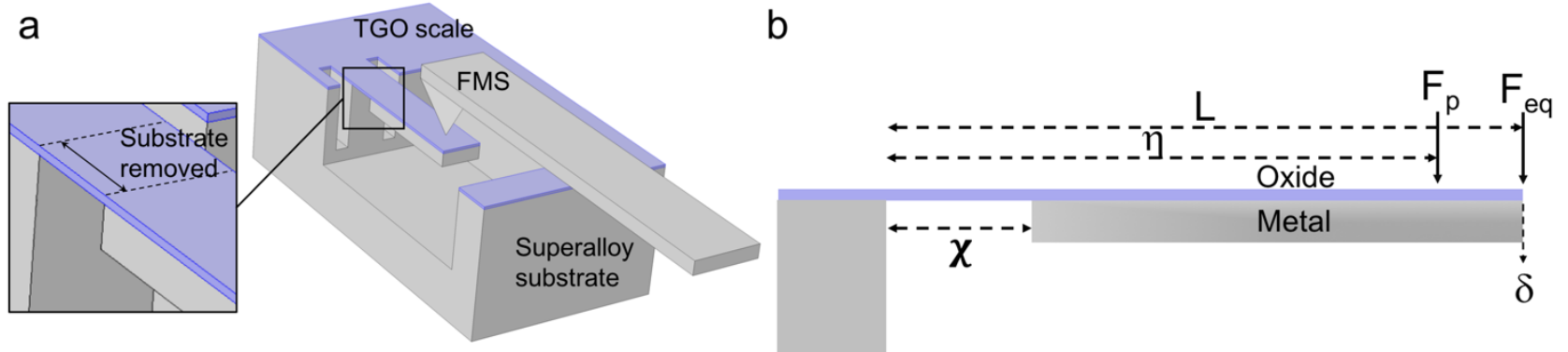

Figure 2: (a) Experimental setup for micro-cantilever bending tests. The oxide layer is shown in purple and the metal in grey. The oxide layer is isolated from the metal towards the fixed end. (b) Schematic of cantilever geometry with relevant notation.

As the FMS tip is made of silicon, it does not indent the surface of the cantilever. Instead the tip slides along the oxide surface with increasing displacements (as seen from Fig. 3a and b), changing the effective span length, $\eta$. In order to allow representation of the bending tests in terms of force and displacements, we chose to use an equivalent force, $F_{e q}=F_{p} \cdot(\eta / L)$, which represents a force applied to the free end of the cantilever resulting in the same bending moment as the actual force applied $\left(F_{p}\right)$, and the free end displacement. Figure $3 \mathrm{~d}$ shows a representative example of such force-displacement curves for two consecutive loading cycles. While the initial response is linear, as expected, a nonlinear behavior is observed at loads above approximately $4 \mu \mathrm{N}$. This behavior is observed in all cantilevers, but the force and displacement levels where the transition occurs varies. A non-linear response at large displacements during micro-cantilever bending is not necessarily an indication of plastic deformation. There are several other factors leading to deviations from linearity, in particular a horizontal force component ( $F_{x}$ in Fig. $3 b$ ), which develops as the angle, $\theta$, between the cantilever and the horizontal plane increases during bending [20]. This can be corrected for analytically by a factor $(\tan \theta / \theta)^{1 / 2}$ [20], but the correction amounts to around $13 \%$ at an angle of $45^{\circ}$, which is not enough to obtain a fully linear response at higher loads in the present case, indicating that there are other contributing factors.

Table 1: Data for cantilever experiments. $L$ - Total cantilever length, $w$ - width, $h-$ thickness of isolated oxide, $\chi$ - length of isolated oxide, $\delta_{r}$ - residual displacement and $\delta_{\max }$ - maximum displacement during testing. The uncertainties in the cantilever dimensions are based on combining the uncertainty in the measurement method with the observed variations occurring within a cantilever.

\begin{tabular}{|c|c|c|c|c|c|c|}
\hline Cantilever & $\boldsymbol{L}(\boldsymbol{\mu} \mathbf{m})$ & $\boldsymbol{w}(\boldsymbol{\mu} \mathbf{m})$ & $\boldsymbol{h}(\boldsymbol{\mu m})$ & $\chi(\boldsymbol{\mu m})$ & $\boldsymbol{\delta}_{\boldsymbol{r}}(\boldsymbol{\mu \mathbf { m } )}$ & $\boldsymbol{\delta}_{\max }(\boldsymbol{\mu m})$ \\
\hline $\mathbf{A}$ & $11.05 \pm 0.03$ & $2.66 \pm 0.03$ & $0.19 \pm 0.02$ & $2.81 \pm 0.04$ & $1.14 \pm 0.03$ & $7.92 \pm 0.04$ \\
\hline $\mathbf{B}$ & $13.26 \pm 0.07$ & $2.82 \pm 0.03$ & $0.23 \pm 0.02$ & $2.28 \pm 0.01$ & $1.75 \pm 0.06$ & $8.43 \pm 0.07$ \\
\hline $\mathbf{C}$ & $11.52 \pm 0.08$ & $2.27 \pm 0.03$ & $0.21 \pm 0.02$ & $2.71 \pm 0.02$ & $1.76 \pm 0.06$ & $7.53 \pm 0.05$ \\
\hline $\mathbf{D}$ & $11.50 \pm 0.04$ & $3.00 \pm 0.03$ & $0.23 \pm 0.02$ & $2.52 \pm 0.02$ & $1.01 \pm 0.04$ & $6.93 \pm 0.03$ \\
\hline
\end{tabular}



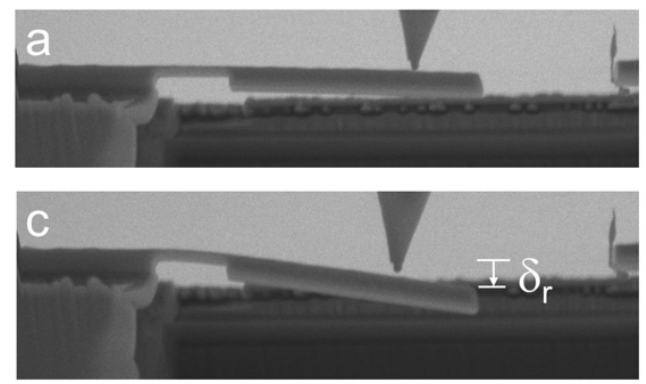
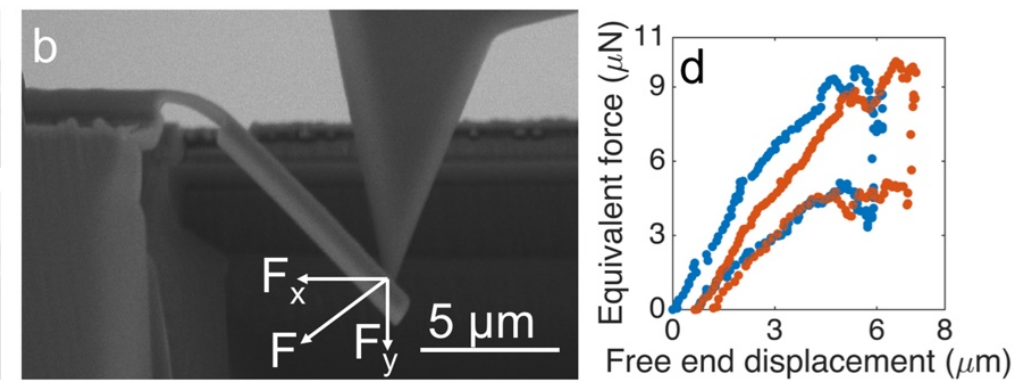

Figure 3: Example of a micro-cantilever bending test (cantilever C). (a) Cantilever before testing; (b) at point of maximum deflection; and (c) residual displacement indicating plasticity after test. $F_{x}$ and $F_{y}$ in (b) are the horizontal and vertical force component, respectively. (d) Representative equivalent force-free end displacement curves for two consecutive loading cycles.

Another prominent feature of the force-displacement curves in Fig. 3d is the force oscillations in the non-linear region, which are thought to be related to the slipping of the FMS tip over the rough oxide surface. After load reversal, there is a large drop in the measured force (occurring in all tests to varying degree), after which the unloading response is non-linear, following a similar path as the loading branch of the curve but with lower forces and stiffness. The non-linearity and presence of oscillations also during unloading again points to slipping of the FMS as the underlying reason. Reverse slipping does not, however, explain the large drop in force or subsequent lower force levels during unloading. The most likely explanation for this is non-linearities in the voltage-displacement relationship of the FMS system when the tip slides back up the oxide surface, resulting from the large angle at which the force is applied to the tip giving a non-pure bending of the FMS silicon cantilever. In particular, the rough surface with rather sharp angles could be expected to increase the horizontal force component as the FMS tip is caught in trenches between oxide grains. Upon reloading a cantilever after unloading, the original stiffness is obtained (see Fig. 3d). It should be noted that force drops after load reversals during micro-cantilever bending has been observed previously [21], and was attributed to slipping of the nanoindenter tip also in that case. However, in [21] the subsequent unloading path was parallel to the loading branch, contrary to our observations, which is likely related to the more robust force response and geometry of the nano-indentation system used, as compared to the present micro-manipulator setup. For information purposes the full force-displacements curves for all specimens are included in the supplementary material, but it must be noted that the observed hysteresis loops are not related to the occurrence of plasticity, but rather mainly related to the performance of the FMS system, as described above.

At this point we focus the attention on the displacement response, rather than the forces, and note that there is a residual displacement, $\delta_{r}$, remaining in Fig. $3 \mathrm{c}$ after unloading. Such residual displacements are present in all three cantilevers (see supplementary material), with magnitudes ranging from 1.14 to $1.75 \mu \mathrm{m}$ in magnitude at the free end (Table 1), indicating the occurrence of permanent deformation, which in turn suggests the presence of plastic deformation. As oxide scales are generally assumed to be brittle, in particular at room temperature, a likely mechanism, besides plasticity, which could cause permanent deformation is micro-cracking of the oxide. Formation of a larger crack would result in brittle fracture, but a network of numerous smaller cracks, each being below the critical defect size, could cause permanent pseudo-plastic response. This has been observed 
during small-scale deformation of different nominally brittle ceramic materials [22], where a loss of elastic stiffness was used as an indicator of micro-cracking.

In the present case, we carefully examined the top oxide surfaces by SEM after testing and found no indications of cracking. Nonetheless, as micro-cracks could be expected to close upon unloading, making them difficult to observe, we also compared the stiffness in the linear regions of successive bending cycles and found no conclusive evidence of stiffness reductions. However, since the tests were not initially performed with stiffness measurements in mind, the scatter in the measurements was relatively large. Furthermore, no information regarding the stiffness of the cantilevers after the last bending cycle, after which the largest amount of micro-cracking would be expected, was available. To resolve this, we conducted an additional test (cantilever D in Table 1), where the stiffness was carefully measured before and after the larger bending tests that resulted in a permanent deformation of 1.01 $\mu \mathrm{m}$. Care was taken to ensure that the displacements during the stiffness measurements were small enough to not cause any residual displacements (details are given in supplementary material). The stiffness values before and after large displacement tests were found to be $K_{i}=2.15 \pm 0.11 \mathrm{~N} / \mathrm{m}$ and $K_{f}=2.36 \pm 0.27 \mathrm{~N} / \mathrm{m}$, respectively, implying that there was no significant micro-cracking in the oxide layer and thus favoring the hypothesis of plasticity as the reason behind the permanent deformation. The occurrence of plasticity in the oxide scale is proposed to be achievable through the low probability of defects in the sub- $\mu \mathrm{m}$ structure, allowing sufficiently high tensile stresses for plastic flow to be reached without fracture.

On a final note, it is possible to extract the effective elastic modulus, $E_{\text {eff, }}$, of the composite spinel/chromia scale from the measured stiffness, $K$ (details in supplementary material). Using the measured stiffness for cantilever D, we find $E_{\text {eff }}=194-206 \mathrm{GPa}$. This is consistent with values reported for other spinels (e.g. 198.2 GPa for $\mathrm{NiFe}_{2} \mathrm{O}_{4}$ and $185.7 \mathrm{GPa}$ for $\mathrm{CoFe}_{2} \mathrm{O}_{4}$ [23]), but lower than typical values for $\mathrm{Cr}_{2} \mathrm{O}_{3}$ (e.g. $272 \mathrm{GPa}$ [24]). It should be noted that the accuracy in these measurements is not sufficient to draw any conclusions regarding possible effect of residual strains on the measurement of $E_{\text {eff. }}$ An interesting aspect would be to compare the stiffness during the loading and unloading parts of the same displacement cycle. However, as previously discussed, the limitations of the FMS system during the unloading phase did not permit any reliable information to be extracted in this respect.

However, as pointed out in [20], cantilever bending is not a suitable method for the determination of elastic modulus, as the relative error can easily become very large due to the cubic dependence on the cantilever thickness, $h$. In the present case, $h$ is the most uncertain geometric parameter, as it can vary both along the isolated oxide (which has been accounted for in the estimated error in Table 1) and across the width of the same (which is not considered in the present investigation). Careful measurements of the cantilever geometry using post-test FIB sectioning, preferably in combination with more accurate force measurements, must be undertaken in order to further improve the accuracy of the estimated effective elastic modulus.

In summary, we have proposed a micro-cantilever geometry which provides a residual stressfree isolated thermally grown oxide scale with a thickness in the order of $200 \mathrm{~nm}$, which could be tested in tension through bending. We can conclude that the permanent residual displacement 
repeatedly observed after multiple micro-cantilever bending experiments is caused by plastic deformation of the oxide, rather than micro-cracking. Although further work is necessary to elucidate the detailed mechanisms behind the plastic deformation, the observed plasticity of nominally brittle oxide scales is likely a further example of the size-dependent brittle-to-ductile transition previously discussed. One drawback of the specimen geometry proposed herein is the steep stress gradient in the oxide scale, which subjects only the top-most part of the oxide to high tensile stresses, but further developments are presently under way to address this issue.

\section{Acknowledgements}

This work was carried out at the Swedish High Temperature Corrosion Centre (HTC) at Chalmers University of Technology, funded by the Swedish Energy Authority and GKN Aerospace Engine Systems. Magnus Hörnqvist Colliander acknowledges additional funding from the Swedish Research Council (grant no 2015-04719). Dr. Mohammad Sattari is kindly acknowledged for performing the TEM analysis of the oxide scale.

\section{References}

[1] M. Schütze, Corros. Sci. 35 (1993) 955-963.

[2] H.-J. Christ, Procedia Eng. 55 (2013) 181-190.

[3] L. Viskari, M. Hörnqvist, K.L.L. Moore, Y. Cao, K. Stiller, Acta Mater. 61 (2013) 3630-3639.

[4] H.S. Kitaguchi, H.Y. Li, H.E. Evans, R.G. Ding, I.P. Jones, G. Baxter, P. Bowen, Acta Mater. 61 (2013) 19681981.

[5] S.R.J. Saunders, M.M. Nagl, M. Schütze, Mater. High Temp. 12 (1994) 103-109.

[6] J.R. Nicholls, C. Mendes, P. Hancock, Mater. High Temp. 12 (1994) 85-94.

[7] P. Hancock, J.R. Nicholls, Mater. Sci. Technol. 4 (1988) 398-406.

[8] J. Castaing, A. He, K.P.D. Lagerlöf, A.H. Heuer, Philos. Mag. 84 (2004) 1113-1125.

[9] Y. Zhang, W.W. Gerberich, D.A. Shores, J. Mater. Res. 12 (1997) 697-705.

[10] M. Schütze, Mater. Sci. Technol. 6 (1990) 32-38.

[11] C. Eberl, D.S. Gianola, X. Wang, M.Y. He, A.G. Evans, K.J. Hemker, Acta Mater. 59 (2011) $3612-3620$.

[12] C. Eberl, X. Wang, D.S. Gianola, T.D. Nguyen, M.Y. He, A.G. Evans, K.J. Hemker, J. Am. Ceram. Soc. 94 (2011) $120-127$.

[13] F. Östlund, K. Rzepiejewska-Malyska, K. Leifer, L.M. Hale, Y. Tang, R. Ballarini, W.W. Gerberich, J. Michler, Adv. Funct. Mater. 19 (2009) 2439-2444.

[14] A. Montagne, S. Pathak, X. Maeder, J. Michler, Ceram. Int. 40 (2014) 2083-2090.

[15] S. Korte-Kerzel, MRS Commun. 7 (2017) 109-120.

[16] W.W. Gerberich, J. Michler, W.M. Mook, R. Ghisleni, F. Östlund, D.D. Stauffer, R. Ballarini, J. Mater. Res. 24 (2009) 898-906.

[17] D. Jang, J.R. Greer, Nat. Mater. 9 (2010) 215-219.

[18] Y. Zou, P. Kuczera, A. Sologubenko, T. Sumigawa, T. Kitamura, W. Steurer, R. Spolenak, Nat. Commun. 7 (2016) 12261.

[19] M.M. Nagl, S.R.J. Saunders, W.T. Evans, D.J. Hall, Corros. Sci. 35 (1993) 965-977.

[20] J.-Å. Schweitz, MRS Bull. 17 (1992) 34-45.

[21] T.P. Weihs, S. Hong, J.C. Bravman, W.D. Nix, J. Mater. Res. 3 (1988) 931-942.

[22] A. Pandey, A. Shyam, T.R. Watkins, E. Lara-Curzio, R.J. Stafford, K.J. Hemker, J. Am. Ceram. Soc. 97 (2014) 899-906.

[23] Z. Li, E.S. Fisher, J.Z. Liu, M. V. Nevitt, J. Mater. Sci. 26 (1991) 2621-2624.

[24] P.F. Tortorelli, Le J. Phys. IV 3 (1993) C9-943-C9-949. 


\title{
Room temperature plasticity in thermally grown sub-micron oxide scales revealed by micro-cantilever bending
}

\author{
Supplementary material \\ Anand. H. S. Iyer, Krystyna Stiller, Magnus Hörnqvist Colliander \\ Department of Physics, Chalmers University of Technology \\ 41296-Gothenburg, Sweden
}

\section{A. Details of sample preparation}

The superalloy surface was prepared according to metallographic preparation techniques down to $0.25 \mu \mathrm{m}$ diamond suspension finish prior to exposure. This reduces the influence of surface roughness on oxide growth and results in an oxide layer of rather uniform thickness. The exposure was conducted in an Alumina tube furnace with a hot zone of about $10 \mathrm{~cm}$ (uncertainty in temperature was $\pm 5^{\circ} \mathrm{C}$ ). The sample was placed on an Alumina boat which serves both as a holder and protection from impurities. The furnace consists of a gas inlet through which a regular flow of oxygen at the rate of $250 \mathrm{ml} / \mathrm{min}$ was maintained. Dry oxygen was used for the exposure in order to prevent evaporation of $\mathrm{Cr}_{2} \mathrm{O}_{3}$ in the presence of water vapor. After exposure, FIB cross sectioning was done at various points to determine average thickness of oxide layer.

The oxidized specimens were cut using a low speed saw and the cross section was polished using broad ion beam (BIB) milling in a Leica EM TIC 3X Triple lon Beam cutter system, operated at a voltage of $6.5 \mathrm{kV}$ and a current of $2.4 \mathrm{~mA}$ for 6 hours, in order to get a flat and sharp edge. The micro-cantilevers were prepared through FIB milling. Coarse milling was performed at $30 \mathrm{keV} 30$ $\mathrm{nA}$, and the current was reduced in steps down to $100 \mathrm{pA}$ during subsequent fine milling, in order to achieve a good surface finish and minimum ion implantation.

\section{B. Stiffness measurements on cantilever D}

In order to measure possible changes in the stiffness of cantilever $D$ resulting from microcracking during deformation, five cycles with a maximum equivalent force of around $4 \mu \mathrm{N}$ (corresponding to a stress level in the outer fiber at maximum load of $\sigma=6 F_{\text {eq }} L / w h^{2} \approx 2 \mathrm{GPa}$, assuming linear elastic behavior) were applied before and after the bending experiment. The absence of permanent deformation after elastic cycles was confirmed in the SEM images. Each cycle was performed with a different loading point along the cantilever in order to estimate scatter from evaluation, and the stiffness was evaluated from each data set by a linear fit to the data points from the loading branch of the curves (shown in Fig. S1 and stiffness values for all cycles are given in Table S1).

Table S1. Stiffnesses measured from each elastic loading cycle.

\begin{tabular}{ccc}
\hline & \multicolumn{2}{c}{ Stiffness $(\mathbf{N} / \mathbf{m})$} \\
Cycle & Before bending test & After bending test \\
\hline 1 & 2.15 & 2.19 \\
2 & 1.96 & 2.75 \\
3 & 2.17 & 2.21 \\
4 & 2.19 & 2.52 \\
5 & 2.26 & 2.12 \\
\hline Average & 2.15 & 2.36
\end{tabular}




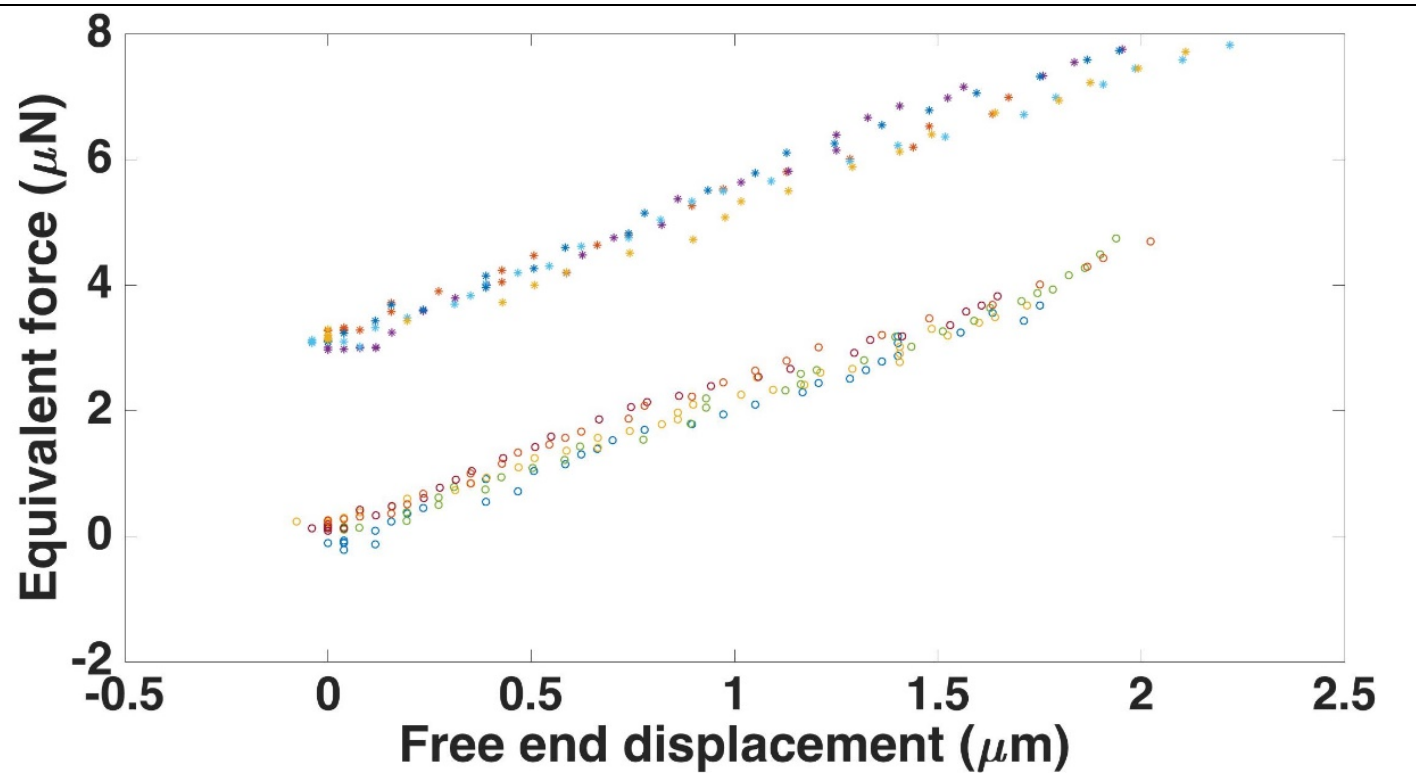

Figure S1: Force-displacement curves from elastic small displacement bending experiments for cantilever D. The 'o' represent before large displacement tests and '*' represent after large displacement tests. The force values for the 'after' has been shifted above by $3 \mu \mathrm{N}$ for clarity.

\section{Geometric factor - Evaluation of elastic modulus.}

The geometry of the cantilever in the experiments can be considered as a combination of the following elementary cases:

a. Cantilever with point load and moment at free end (oxide).

b. Cantilever with point load at the free end (oxide with substrate).

a

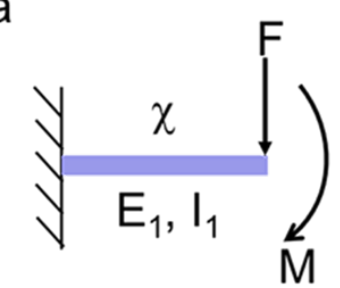

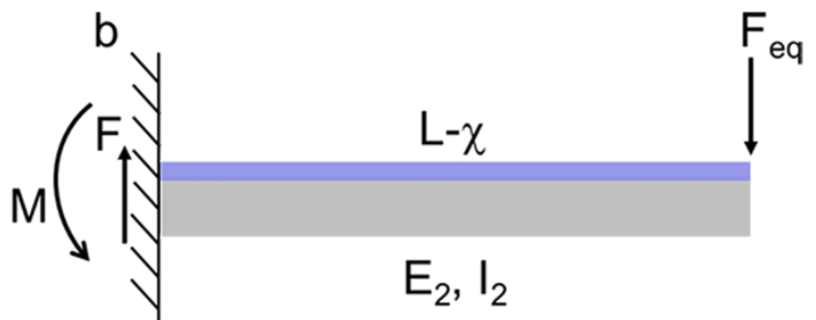

$\theta_{1}$

C

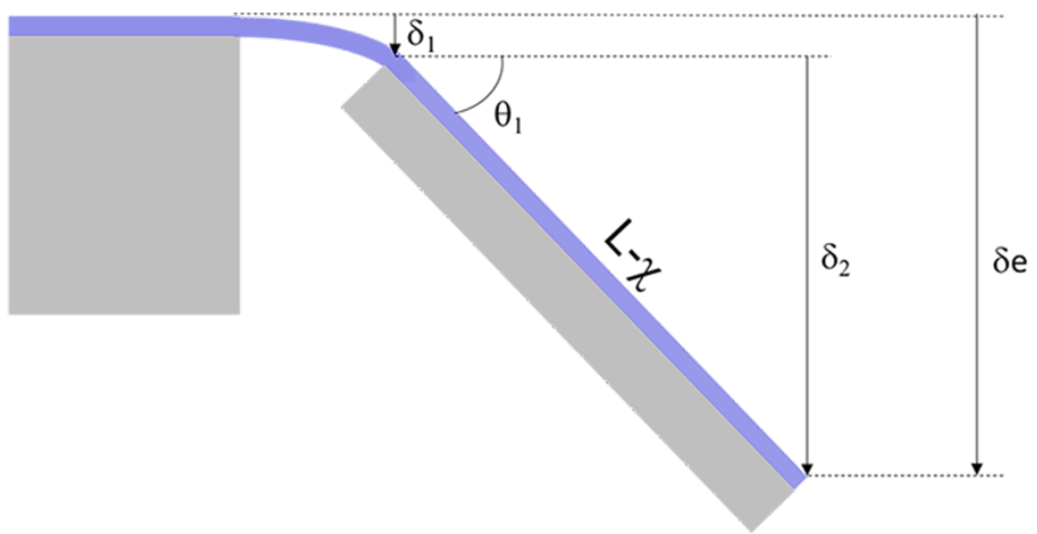


Figure S2: Schematic of cantilever. a) Cantilever with point load and moment (oxide), b) cantilever with a point load (oxide with metal), c) displacements. Note that $E$ is the elastic modulus and $I$ is the moment of inertia.

The following assumptions are made for the calculations:

1. $\theta_{1}$, which is the slope at the end of the pure oxide part, is the small and hence $\sin \theta_{1} \approx \theta_{1}$. This is justified by the measured maximum slope during the elastic cycles, which is in the order of $10^{\circ}(\sin \theta / \theta=0.995)$.

2. The part of the cantilever with underlying metal substrate $(L-\chi)$ remains straight during bending. This is motivated by $I_{2} \gg I_{1}$ (a function and the cube of the thickness), and was verified from the SEM images.

From figure S2(c) we have,

$\delta_{2}=\sin \theta_{1}(L-\chi) \approx \theta_{1}(L-\chi)$

The slope, $\theta_{1}$, is given by the sum of the slopes caused by the applied point load and bending moment. Using the standard formulae, we get,

$\theta_{1}=\frac{F_{e q} \chi^{2}}{2 E_{1} I_{1}}+\frac{F_{e q}(L-\chi) \chi}{E_{1} I_{1}}$

Similarly, the displacement for the oxide part is given by

$\delta_{1}=\frac{F_{e q} \chi^{3}}{3 E_{1} I_{1}}+\frac{F_{e q}(L-\chi) \chi^{2}}{2 E_{1} I_{1}}$

Combining the equations (1) $-(3)$, the total displacement, $\delta_{\mathrm{e}}=\delta_{1}+\delta_{2}$, can then be found from,

$\delta_{e}=\frac{F_{e q} g}{E_{1} I_{1}}$

where the geometric factor, $g$, is given by

$g=L^{2} \chi-L \chi^{2}+\frac{\chi^{3}}{3}$

The above equation for displacement can be used to calculate the elastic modulus, $E$ of the material. Rewriting the equation, we get,

$E=g \frac{K}{I}$

where, $K=F_{e q} / \delta_{e}$, the stiffness measured from elastic part of force-displacement curves, $l$ is the area moment of inertia of the cross section $\left(I=w h^{3} / 12\right)$ and $g$ is the above geometric factor.

The above model neglects a number of characteristics of the actual micro-cantilever. Besides the assumed absence of deformation of the oxide+metal part of the cantilever, the most important ones are the boundary conditions applied to the two elementary cases, assuming rigidly fixed, rather than constrained, clamping. The bases of both elementary case cantilevers will 
undergo limited rotation, and the supporting metal can deform under the oxide cantilever. In order to assess the accuracy of the analytical model presented above, we compared the results with linear elastic 3D finite element (FE) simulations performed in COMSOL Multiphysics 5.0. The simulated cantilever, shown in Fig. S3, had the following dimensions: $L=10 \mu \mathrm{m}, w=3 \mu \mathrm{m}, h=0.2 \mu \mathrm{m}$, $\chi=2.5 \mu \mathrm{m}$ and $H=0.6 \mu \mathrm{m}$. The load was distributed along the end of the cantilever and the resulting displacements were extracted. Only half the cantilever was simulated due to symmetry along the central plane.

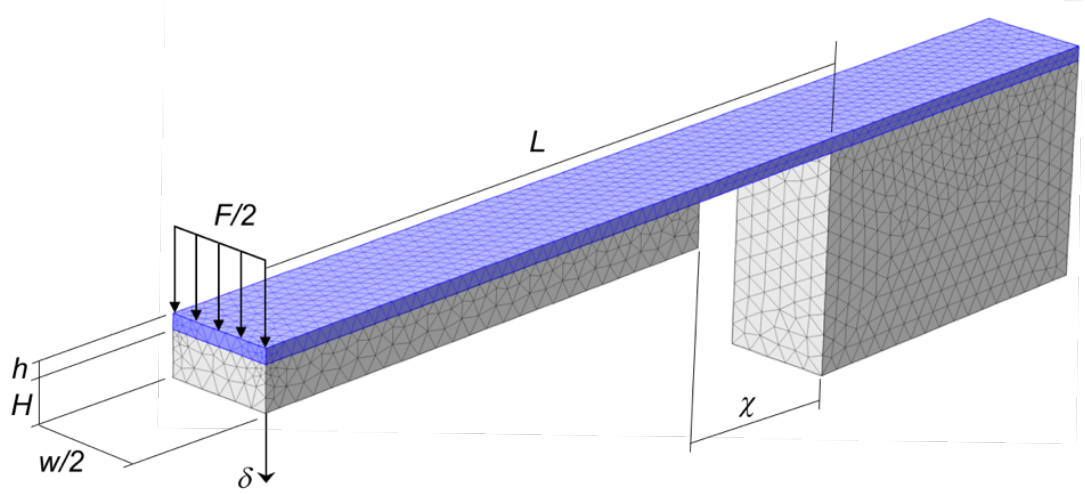

Figure S3: Finite element model used for assessment of analytical model accuracy.

The properties of the metal were $E_{m}=200 \mathrm{GPa}$ and $v_{m}=0.3$, whereas the elastic modulus of the oxide, $E_{o x}$, was varied ( $v_{o x}$ was kept constant at 0.25 ). Three cases were simulated: (i) a compliant oxide, $E_{o x} / E_{m}=0.75$; (ii) a neutral oxide, $E_{o x} / E_{m}=1.0$; and (iii) a stiff oxide, $E_{o x} / E_{m}=1.5$. The resulting force-displacement curves can be seen in Fig. S4, together with the predictions from Eq. (5). The analytical model is slightly stiffer than the FE model, but the difference is small. Comparing the stiffnesses from the FE model $\left(K_{F E}\right)$ with the analytical values ( $K_{a}$ according to Eq. (6)), we find that $K_{a} / K_{F E}=1.043,1.051$ and 1.066 for the compliant, neutral, and stiff oxide, respectively. Consequently, if Eq. (6) is used to extract the elastic modulus we get under-estimation of the actual values of $E_{o x}$ by $4.1 \%, 4.9 \%$ and $6.2 \%$, for the three different cases. Considering that the elastic modulus found in the present study is close to that of the superalloy substrate, we can expect an under-estimation of the actual modulus of around $5 \%$ (assuming perfect geometry). Given that the error from uncertainties in the geometry is expected to be considerably larger than this, the analytical model gives satisfactory accuracy for approximately evaluating the elastic modulus.

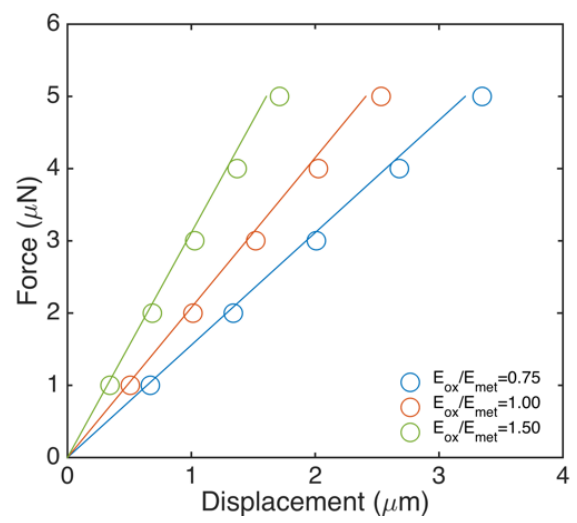

Figure S4: Results from finite element simulations (circles) and analytical model in Eq. (5) (solid lines) for three different relative stiffnesses of oxide and metal. 


\section{SEM images for cantilevers A, B and D}

The SEM images for cantilevers A, B and D have been shown below in order to show the presence of permanent deformation after all bending tests and confirm the repeatability of the experiment.
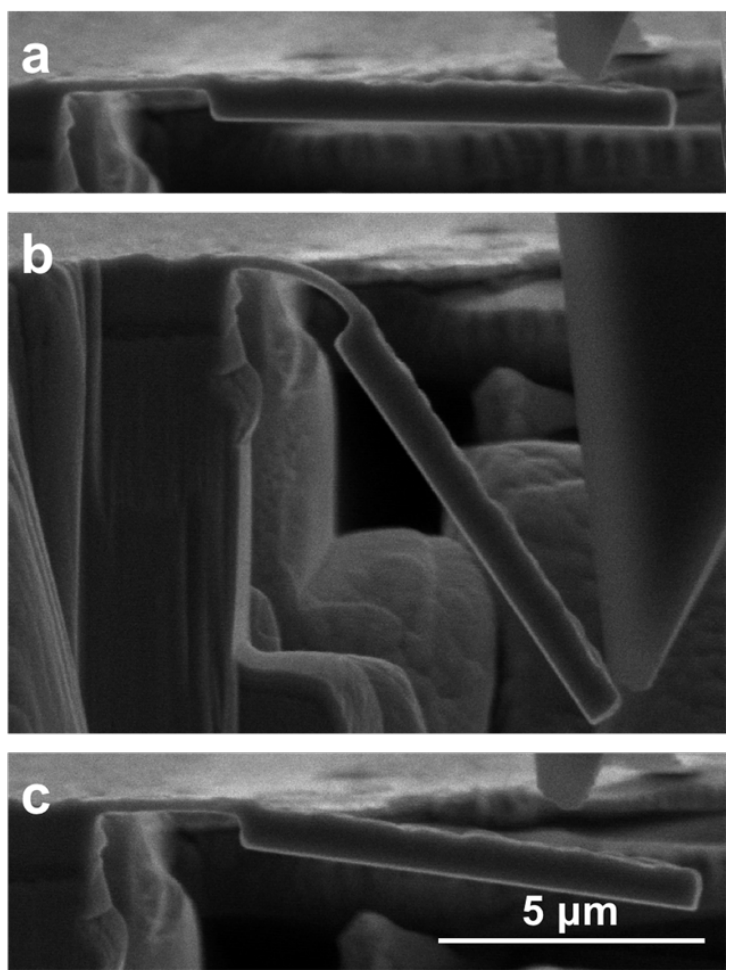

Figure S5: SEM images from bending test for cantilever A. a) before, b) max displacement and c) after.
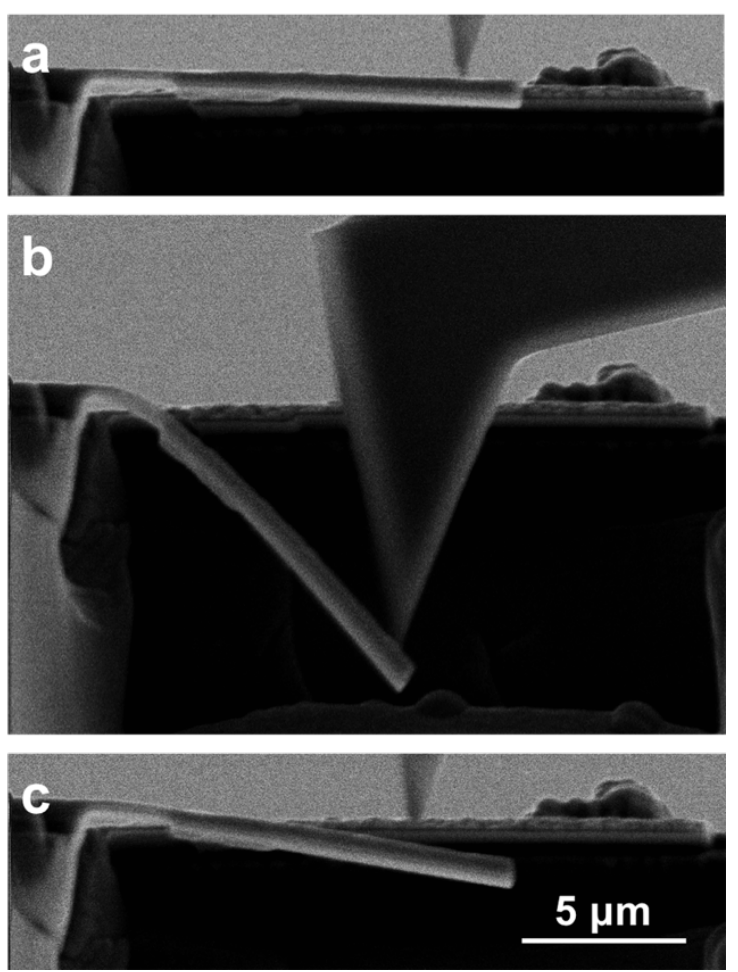

Figure S6: SEM images from bending tests for cantilever B. a) before, b) max displacement and c) after. 

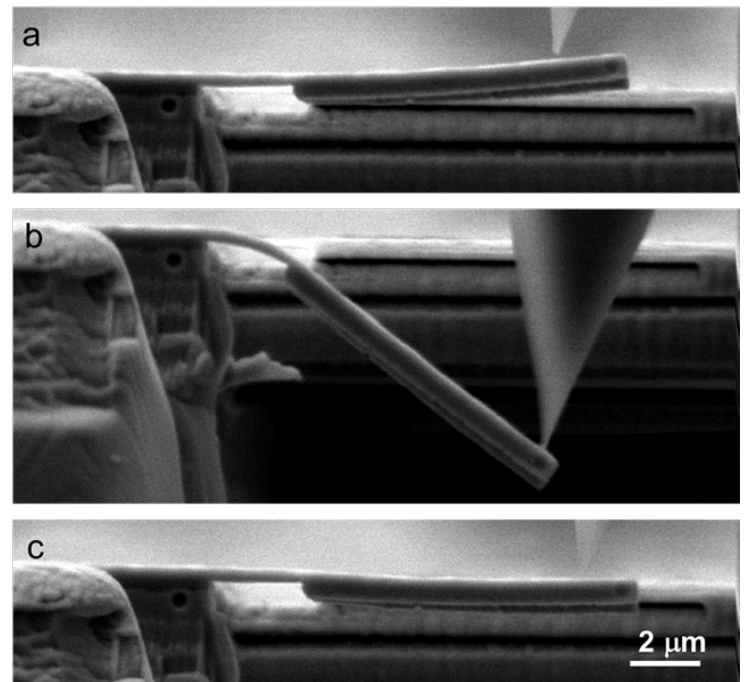

Figure S7: SEM images from bending tests for cantilever $D$. a) before, b) max displacement and c) after.

\section{E. Force-displacement curves}

(a)

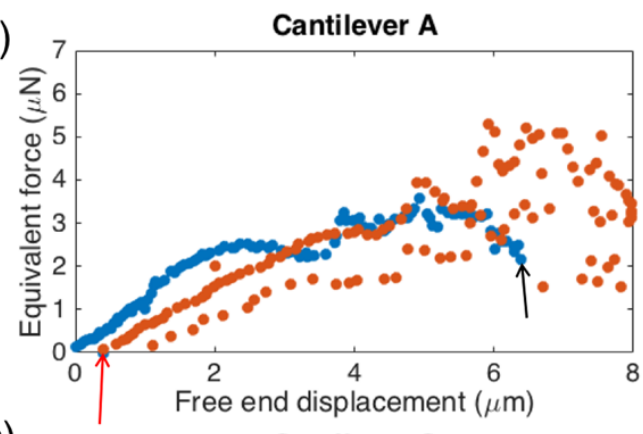

(c)

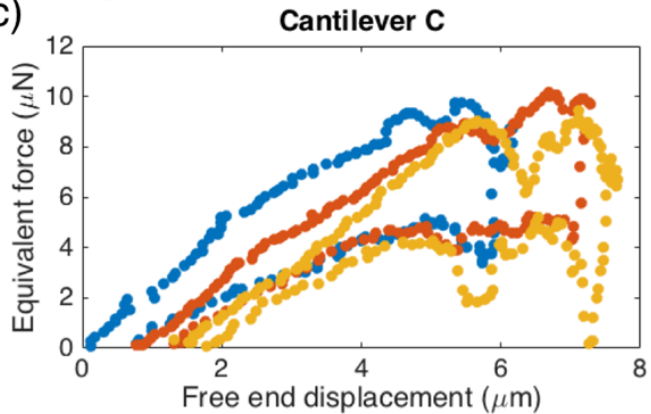

(b)

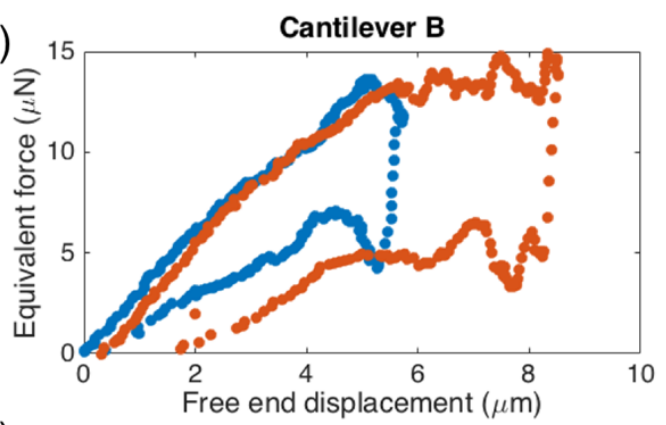

(d)

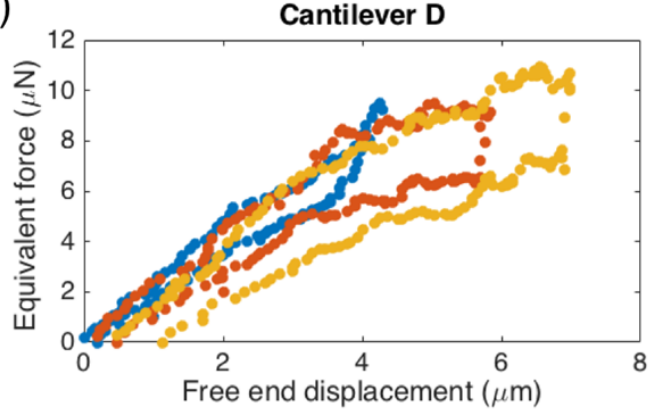

Figure S8: Force displacement curves for all four cantilevers; (a) Two loading cycles shown for cantilever A. In this test, the FMS system stopped recording during the first loading cycle, at the point indicated by the black arrow. The red arrow shows the residual displacement for the corresponding loading cycle, measured from SEM images after unloading. (b) Two loading cycles shown for cantilever B; (c) and (d) three loading cycles shown for cantilever C and D, respectively. 